\title{
The Principle of Minimal Potential Energy of Mixed Variables to Solve the \\ Bending of Cantilever Rectangular Plate under Uniform Load
}

\author{
Liu Xin-min ${ }^{1, a}$, JiangJia-heng ${ }^{2, b}$, DongJing-bo ${ }^{3, c}$ and GuoDun ${ }^{4, d}$ \\ ${ }^{1}$ YanShan University, college of civil engineering and mechanics, Hebei Qinhuangdao China \\ ${ }^{2}$ YanShan University, college of civil engineering and mechanics, Hebei Qinhuangdao ,China \\ ${ }^{3}$ YanShan University, college of civil engineering and mechanics, Hebei Qinhuangdao ,China

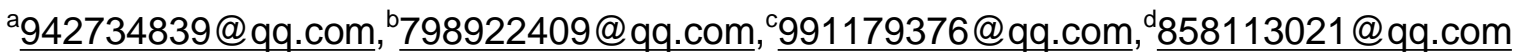

Keywords: Mixed variable minimum potential energy principle; Deflection surface equation; Numeral Calculations; Cantilever rectangular thin plate;

Abstract. The problem of the balance of the cantilever rectangular thin plate under the uniform load is solved by using the minimum potential energy of mixed variables. The solution process is clear, and the deflection surface equation is given. Through the numerical calculation, the calculation results of the graph form are obtained, which are compared with the finite element results, and the accuracy of the numerical results is verified. It shows that the method presented in this paper has certain practical significance to the practical application of engineering, which can be directly applied to the actual project.

\section{Introduction}

With the development of the national economy, the construction industry is developing rapidly. Many construction projects involve the balance problems about a variety of elastic sheet and plates.The principle of energy gradually receives people's attention and is more and more popular in practical engineering applications because of its universality, simplicity and accuracy in solving various problems in practical engineering problems. However, for the present, some of the existing research methods can not meet the design requirements. In this paper, the balance of the cantilever rectangular thin plate is further studied by the minimum potential energy principle of mixed variables given in [1]. The numerical solution of the bending problem of the rectangular thin plate with hanging corners, one fixed edge and three free edges under the uniform load is given , and compared with the finite calculation results, and the results can be directly applied to engineering design.

\section{The Minimum Potential Energy Principle of Mixed Variables for Curved Rectangular Thin} Plate

Consider the bending of a free rectangular plate with two adjacent edges fixed under the distributed load, as shown in Figure 1(a). Release the fixed bending restraints at both ends, substitute the distribution bending moments $\bar{M}_{x_{0}}$ and $\bar{M}_{y 0}$, respectively. The free edge deflection curve equations are denoted as $\bar{w}_{x a}$ and $\bar{w}_{y b}$, respectively, and the suspension corner deflection is $\bar{w}_{a b}$, as shown in Figure 1(b). 


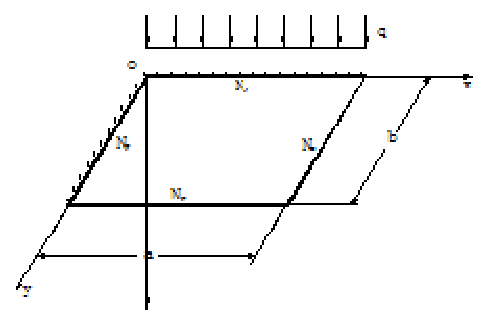

(a)

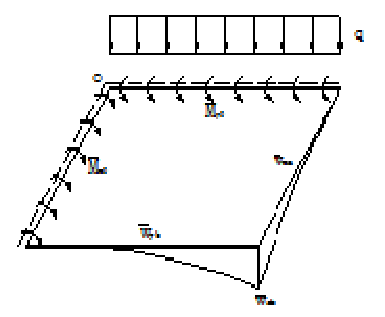

(b)

Fig. 1 The bending rectangular plate with two adjacentclamped edges and the other two free edges under uniform load

The minimum potential energy principle of the mixed variable is applied to the total potential energy of the rectangular slab in the graph:

$$
\begin{aligned}
\Pi_{m p}= & \int_{0}^{a} \int_{0}^{b} \frac{1}{2} D\left\{\left(\frac{\partial^{2} w}{\partial x^{2}}+\frac{\partial^{2} w}{\partial y^{2}}\right)^{2}-2(1-v)\left[\frac{\partial^{2} w}{\partial x^{2}} \frac{\partial^{2} w}{\partial y^{2}}-\left(\frac{\partial^{2} w}{\partial x \partial y}\right)^{2}\right]\right\} d x d y- \\
& \int_{0}^{a} \int_{0}^{b} q w d x d y \int_{0}^{b} \bar{M}_{x 0}\left(\frac{\partial w}{\partial x}\right)_{x=0} d y-\int_{0}^{a} \bar{M}_{y 0}\left(\frac{\partial w}{\partial y}\right)_{y=0} d x+\int_{0}^{b} \bar{w}_{x a}\left(V_{x}\right)_{x=a} d y+\int_{0}^{a} \bar{w}_{y b}\left(V_{y}\right)_{y=b} d x-\bar{w}_{a b} R_{a b}
\end{aligned}
$$

The upper part is called the total potential energy of the minimum potential energy of the mixed variable, or the combined total potential energy.

In the case of (1),take the variational extremes of $w, V_{x a}, V_{y b}$ and $R_{a b}$, and apply the zero-variational principle of the inner boundary and the basic preparatory theorem of the variational method, the Euler equation and the natural boundary condition are obtained respectively :

$$
\begin{array}{lll}
D\left(\frac{\partial^{4} w}{\partial x^{4}}+2 \frac{\partial^{4} w}{\partial x^{2} \partial y^{2}}+\frac{\partial^{4} w}{\partial y^{4}}\right)=q & (2) & -D\left(\frac{\partial^{2} w}{\partial x^{2}}+v \frac{\partial^{2} w}{\partial y^{2}}\right)_{x=0}=\bar{M}_{x 0} \\
-D\left(\frac{\partial^{2} w}{\partial x^{2}}+v \frac{\partial^{2} w}{\partial y^{2}}\right)_{x=a}=0 & (4) & -D\left(\frac{\partial^{2} w}{\partial y^{2}}+v \frac{\partial^{2} w}{\partial x^{2}}\right)_{y=0}=\bar{M}_{y 0} \\
-D\left(\frac{\partial^{2} w}{\partial y^{2}}+v \frac{\partial^{2} w}{\partial x^{2}}\right)_{y=b}=0 & (6) & \left(w_{x}\right)_{x=0}=0 \\
\left(w_{x}\right)_{x=a}-\bar{w}_{x a}=0 & (8) & \left(w_{y}\right)_{y=0}=0 \\
\left(w_{y}\right)_{y=b}-\bar{w}_{y b}=0 & (10) & \left(w_{x y}\right)_{x=a, y=b}-\bar{w}_{a b}=0 \\
\left(w_{x y}\right)_{x=0, y=0}=\left(w_{x y}\right)_{x=a, y=0}=\left(w_{x y}\right)_{x=a, y=b}=0 &
\end{array}
$$

The above is the minimum potential energy principle of the mixed variable of the rectangular plate. 


\section{Cantilever rectangular plate with two hanging corners, one fixed edge and three free edges}

Considering that the rectangular plate is under uniform load, one edge is fixed and three edges are free, based on the previous text.

Assume that the deflection surface equation is.

$$
w(x, y)=w_{1}(x, y)+w_{2}(x, y)+\frac{y}{b} k
$$

among them, $w_{1}$ and $w_{2}$ is given in [1].

It is assumed firstly in the calculation of the rectangular plate with the one edge fixed and the other three edges free.

$$
\begin{aligned}
\bar{w}_{x 0} & =\bar{w}_{x a}=\sum_{n=1,2}^{\infty} a_{n} \sin \beta_{n} y+\frac{y}{b} k \quad \text { (14) } \quad \bar{w}_{y b}=\sum_{m=1,2}^{\infty} d_{m} \sin \alpha_{m} x+k \\
\bar{M}_{y 0} & =\sum_{m=1,2}^{\infty} C_{m} \sin \alpha_{m} x
\end{aligned}
$$

And $\mathrm{k}$ is the deflection of the free point.

Apply $w_{1}$ and $w_{2}$ into the case of (1) and take the variational extremes of $w, V_{x 0}, V_{x a}, V_{y 0}$, $V_{v b}$ and $R_{a b}$,simplify it,and according to the basic preparatory theorem of the variational method, the Euler equation and the natural boundary condition are obtained respectively :

$$
\begin{gathered}
D\left(\frac{\partial^{4} w_{1}}{\partial x^{4}}+2 \frac{\partial^{4} w_{1}}{\partial x^{2} \partial y^{2}}+\frac{\partial^{4} w_{1}}{\partial y^{4}}\right)=\frac{q}{2} \\
-D\left(\frac{\partial^{2} w_{1}}{\partial x^{2}}+v \frac{\partial^{2} w_{1}}{\partial y^{2}}\right)_{x=0}=0 \\
-D\left(\frac{\partial^{2} w_{2}}{\partial y^{2}}+v \frac{\partial^{2} w_{2}}{\partial x^{2}}\right)_{y=0}=\bar{M}_{y 0} \\
w_{1 x 0}=\bar{w}_{x 0} \\
w_{2 y 0}=0
\end{gathered}
$$$$
D\left(\frac{\partial^{4} w_{2}}{\partial x^{4}}+2 \frac{\partial^{4} w_{2}}{\partial x^{2} \partial y^{2}}+\frac{\partial^{4} w_{2}}{\partial y^{4}}\right)=\frac{q}{2}
$$$$
-D\left(\frac{\partial^{2} w_{1}}{\partial x^{2}}+v \frac{\partial^{2} w_{1}}{\partial y^{2}}\right)_{x=a}=0
$$$$
-D\left(\frac{\partial^{2} w_{2}}{\partial y^{2}}+v \frac{\partial^{2} w_{2}}{\partial x^{2}}\right)_{y=b}=0
$$$$
w_{1 x a}-\bar{w}_{x a}=0
$$$$
w_{2 y b}-\bar{w}_{y b}=0
$$

substitute $w_{1}, w_{2}$ into $(17) \sim(26)$, calculate and simplify to get the deflection surface equation:

$$
w(x, y)=\frac{4 q}{D a} \sum_{m=1,2}^{\infty}\left\{1+\frac{1}{2 \operatorname{ch} \frac{1}{2} \alpha_{m} b}\left[\alpha_{m}\left(y-\frac{b}{2}\right) \operatorname{sh} \alpha_{m}\left(y-\frac{b}{2}\right)-\left(2+\frac{1}{2} \alpha_{m} b t h \frac{1}{2} \alpha_{m} b\right) \operatorname{ch} \alpha_{m}\left(y-\frac{b}{2}\right)\right]\right\} \frac{\left[1+(-1)^{m+1}\right]^{5}}{\alpha_{m}^{5}} \sin \alpha_{m} x+
$$




$$
\begin{aligned}
& {\left[\operatorname{or} \frac{4 q}{D b} \sum_{n=1,2}^{\infty}\left\{1+\frac{1}{2 \operatorname{ch} \frac{1}{2} \beta_{n} a}\left[\beta_{n}\left(x-\frac{a}{2}\right) \operatorname{sh} \beta_{n}\left(x-\frac{a}{2}\right)-\left(2+\frac{1}{2} \beta_{\mathrm{n}} a \operatorname{th} \frac{1}{2} \beta_{\mathrm{n}} a\right) \operatorname{ch} \beta_{\mathrm{n}}\left(x-\frac{a}{2}\right)\right]\right\} \frac{\left[1+(-1)^{n+1}\right]_{n}}{\beta_{n}^{5}} \sin \beta_{\mathrm{n}} y+\right]} \\
& \frac{1}{2 D} \sum_{m=1,3}^{\infty}\left(-\frac{\alpha_{m} b}{\operatorname{sh}^{2} \alpha_{m} b} \operatorname{sh} \alpha_{m} y+\operatorname{cth} \alpha_{m} b \alpha_{m} y \operatorname{ch} \alpha_{m} y-\alpha_{m} y \operatorname{sh} \alpha_{m} y\right) \frac{C_{m}}{\alpha_{m}^{2}} \sin \alpha_{m} x+ \\
& \frac{1}{2} \sum_{n=1,2}^{\infty}\left\{2\left(\operatorname{ch} \beta_{n} x-\operatorname{th} \frac{1}{2} \beta_{n} a \operatorname{sh} \beta_{n} x\right)+(1-\mu)\left[\operatorname{th} \frac{1}{2} \beta_{n} a \cdot\left(\frac{\beta_{n} a}{\operatorname{sh} \beta_{n} a} \operatorname{sh} \beta_{n} x+\beta_{n} x \operatorname{ch} \beta_{n} x\right)-\beta_{n} x \operatorname{sh} \beta_{n} x\right]\right\} a_{n} \sin \beta_{n} y+ \\
& \frac{1}{2} \sum_{m=1,3}^{\infty}\left[2+(1-\mu)\left(\alpha_{m} b \operatorname{cth} \alpha_{m} b-\alpha_{m} y \operatorname{cth} \alpha_{m} y\right)\right] \frac{d_{m}}{\operatorname{sh} \alpha_{m} b} \operatorname{sh} \alpha_{m} y \sin \alpha_{m} x+\frac{y}{b} k
\end{aligned}
$$

The boundary conditions to be satisfied in this paper are:

$$
\begin{array}{ll}
\left(\frac{\partial w}{\partial y}\right)_{y=0}=0 & \text { (28) }-D\left[\frac{\partial^{3} w}{\partial x^{3}}+(2-\mu) \frac{\partial^{3} w}{\partial x \partial y^{2}}\right]_{x=0, a}=0 \\
-D\left[\frac{\partial^{3} w}{\partial y^{3}}+(2-\mu) \frac{\partial^{3} w}{\partial x^{2} \partial y}\right]_{y=b}=0 & (30)-2 D(1-\mu)\left(\frac{\partial^{2} w}{\partial x \partial y}\right)_{x=a, y=b}=0
\end{array}
$$

1)

The deflection surface equation(27) is substituted into the above boundary conditions (28) (31), and then the corresponding execution equations of the boundary conditions of the rectangular plate under the boundary condition are obtained as follows:

$$
\begin{aligned}
& \frac{2 q}{D b}\left(\operatorname{th} \frac{1}{2} \alpha_{m} b-\frac{\alpha_{m} b}{2 \operatorname{ch}^{2} \frac{1}{2} \alpha_{m} b}\right) \frac{1}{\alpha_{m}^{4}}+\frac{1}{2 D}\left(\operatorname{ch} \alpha_{m} b-\frac{\alpha_{m} b}{\operatorname{sh} \alpha_{m} b}\right) \frac{C_{m}}{\alpha_{m} \operatorname{sh} \alpha_{m} b}+\frac{1}{2}\left[1+\alpha_{m} b \operatorname{cth} \alpha_{m} b+\mu\left(1-\alpha_{m} b \operatorname{cth} \alpha_{m} b\right)\right] \frac{\alpha_{m} d_{m}}{\operatorname{sh} \alpha_{m} b}+ \\
& \frac{2}{a} \sum_{n=1,2}^{\infty} \frac{\alpha_{m}}{K_{m n}^{2}}\left[\alpha_{m}^{2} \beta_{n}+(2-\mu) \beta_{n}^{3}\right] a_{n}+\frac{4}{a b} \frac{1}{\alpha_{m}} k=0 \\
& \frac{q}{b}\left[1+(-1)^{n+1}\right] .\left[(3-\mu) \operatorname{th} \frac{1}{2} \beta_{n} a-(1-\mu) \frac{\beta_{n} a}{2 \operatorname{ch}^{2} \frac{1}{2} \beta_{n} a}\right] \frac{1}{\beta_{n}^{2}}+\frac{1}{2}\left[\left(1+\beta_{n} a \operatorname{cth} \beta_{n} a\right)+\mu\left(1-\beta_{n} a \operatorname{cth} \beta_{n} a\right)\right] \frac{\beta_{n}}{\operatorname{sh} \beta_{n} a} A_{n}+ \\
& \frac{2}{b} \sum_{m=1,3}^{\infty} \frac{\alpha_{m}}{K_{m n}^{2}}\left[\alpha_{m}^{2} \beta_{n}+(2-\mu) \beta_{n}^{3}\right] C_{m}+\frac{D}{2}\left[-2\left(1-\mu^{2}\right)+(1-\mu)^{2}\left(\frac{\beta_{n} a}{\operatorname{sh} \beta_{n} a}-1\right)\right] \beta_{n}^{3} \operatorname{th} \frac{1}{2} \beta_{n} a a_{n}- \\
& \frac{2 q}{b}\left[-(3-\mu) \operatorname{th} \frac{1}{2} \alpha_{m} b+(1-\mu) \frac{\alpha_{m} b}{2 \operatorname{ch}^{2} \frac{1}{2} \alpha_{m} b}\right] \frac{1}{\alpha_{m}^{2}}-\frac{1}{2}\left[\left(1+\alpha_{m} b \operatorname{cth} \alpha_{m} b\right)+\mu\left(1-\alpha_{m} b \operatorname{cth} \alpha_{m} b\right)\right] \frac{\alpha_{m}}{\operatorname{sh}_{m} b} C_{m}+
\end{aligned}
$$




$$
\begin{aligned}
& \frac{4 D}{a}(1-\mu)^{2} \sum_{n=1,2}^{\infty} \frac{(-1)^{n} \alpha_{m}^{3} \beta_{n}^{3}}{K_{m n}^{2}} a_{n}+\frac{D}{2}\left[2\left(1-\mu^{2}\right) \operatorname{ch} \alpha_{m} b+(1-\mu)^{2}\left(\operatorname{ch} \alpha_{m} b+\frac{1}{\operatorname{sh} \alpha_{m} b}\right)\right] \frac{\alpha_{m}^{3}}{\operatorname{sh} \alpha_{m} b} d_{m}=0 \\
& -4(1-\mu) \frac{q}{a} \sum_{m=1,2}^{\infty}\left(\operatorname{th} \frac{1}{2} \alpha_{m} b-\frac{\alpha_{m} b}{2 \operatorname{ch}^{2} \frac{1}{2} \alpha_{m} b}\right) \frac{1}{\alpha_{m}^{3}}+(1-\mu) \sum_{m=1,3}^{\infty}\left(1-\alpha_{m} b \operatorname{cth} \alpha_{m} b\right) \frac{1}{\operatorname{sh} \alpha_{m} b} C_{m}+ \\
& D(1-\mu) \sum_{n=1,2}^{\infty}\left[\left(\frac{\beta_{n} a}{\operatorname{sh} \beta_{n} a}-1\right)-\mu\left(\frac{\beta_{n} a}{\operatorname{sh} \beta_{n} a}+1\right)\right] \operatorname{th} \frac{1}{2} \beta_{\mathrm{n}} a \beta_{n}^{2}(-1)^{n} a_{n}+D(1-\mu) \sum_{m=1,3}^{\infty}\left[\left(\operatorname{ch} \alpha_{m} b+\frac{\alpha_{m} b}{\operatorname{sh} \alpha_{m} b}\right)+\mu\left(\operatorname{ch} \alpha_{m} b-\frac{\alpha_{m} b}{\operatorname{sh} \alpha_{m} b}\right)\right] \frac{\alpha_{m}^{2}}{\operatorname{sh} \alpha_{m} b} d_{m}=0
\end{aligned}
$$

\section{Numeral Calculations}

As a numerical example,take the square sheet of $a=b=1, \mathrm{H}=1, \mu=0.3$, and $a_{n}=b_{n}, d_{m}$. This example contains four unknown parameters $C_{m} 、 a_{n} 、 d_{m}, \mathrm{k}$, And the iterative improvement method is used to solve it.Using Matlab software programming to calculate the available parameters $A_{n}, C_{m}, b_{n}, d_{m}, \mathrm{k}$.

Table 1 Table of deflection values at $y=b \quad\left(q a^{4} / D\right) \times 10^{-1}$

\begin{tabular}{c|c|c|c|c|c|c|c|c|c|c}
\hline \multicolumn{2}{c|}{$x / a$} & 0.05 & 0.15 & 0.25 & 0.35 & 0.45 & 0.50 & 0.70 & 0.90 & 1.00 \\
\hline \multirow{3}{*}{$w_{y b}$} & Ansys 值 & 1.290 & 1.291 & 1.294 & 1.296 & 1.297 & 1.297 & 1.296 & 1.291 & 1.289 \\
\cline { 2 - 10 } & 本文 & 1.290 & 1.292 & 1.295 & 1.296 & 1.297 & 1.297 & 1.296 & 1.291 & 1.289 \\
\hline
\end{tabular}

Table 2 Table of deflection values at $x=0\left(q a^{4} / D\right) \times 10^{-1}$

\begin{tabular}{c|c|c|c|c|c|c|c|c|c|c}
\hline \multicolumn{2}{c|}{$y / b$} & 0.1 & 0.2 & 0.3 & 0.4 & 0.5 & 0.6 & 0.7 & 0.8 & 1.0 \\
\hline \multirow{3}{*}{$w_{x 0}$} & Ansys 值 & 0.002 & 0.082 & 0.180 & 0.302 & 0.447 & 0.604 & 0.770 & 0.941 & 1.289 \\
\cline { 2 - 11 } & 本文 & 0.002 & 0.083 & 0.180 & 0.303 & 0.447 & 0.604 & 0.770 & 0.941 & 1.289 \\
\hline
\end{tabular}

Table 3 Calculational results of bending moment at $y=0 \quad\left(-q a^{2}\right)$

\begin{tabular}{c|c|c|c|c|c|c|c|c}
\hline$x / a$ & 0.05 & 0.10 & 0.15 & 0.20 & 0.35 & 0.50 & 0.70 & 0.90 \\
\hline$M_{y 0}$ & 0.4569 & 0.5246 & 0.5326 & 0.5326 & 0.5326 & 0.5325 & 0.5326 & 0.5246 \\
\hline
\end{tabular}

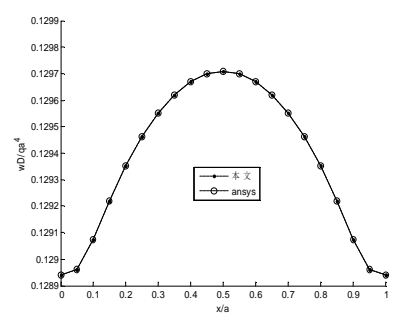

Fig. 2Deflection curves at $y=b$

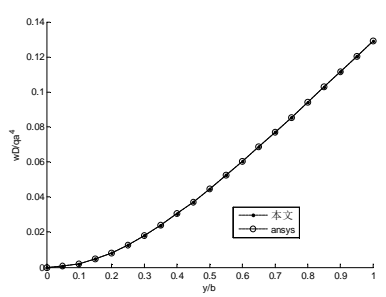

Fig.3 Deflection curves at $x=0$ 


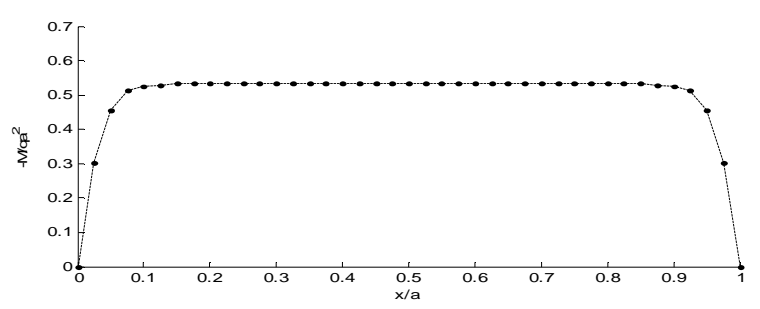

Fig. 4 Graph of bending moment values at $y=0$

The numerical solution of the deflection function is obtained by substituting the results in the deflection surface equation,and the results are shown in Table 1 and Table 2. Table 1 and Table 2 gives the numerical solution of the deflection changing along $x$ and $\mathrm{y}$ for the free edge $\mathrm{y}=\mathrm{b}$ and $\mathrm{x}=0$, Figure 2 and Figure 3 is for the distribution curve of the deflection for the free edge $y=b$ and $x=0$.

Substitute $C_{m}$ into(14)and(16), and we can get the distribution value along $x$ about the bending moment of the fixed edge $y=0$,and the results are shown in Table 3.Figure 4 is for the distribution curve of the bending moment for the fixed edge $y=0$.

The finite element software ANSYS is used to analyze the cantilever rectangular plate. Taking the example: length is $a=b=1$, thickness is $t=0.005$, elastic modulus $E=210 \mathrm{Gpa}$, uniform $\operatorname{load} q=1 \mathrm{~N} / \mathrm{m}^{2}$. The 4-node shell unit is chosen to convert the computer results into a dimensionless quantity. Considering the bending stiffness of the sheet is $D=E t^{3} /\left[12\left(1-v^{2}\right)\right]=1230000 N / m$, and this result is consistent with the result of the minimum potential energy principle of the mixed variable. The results are shown in Table 1 and Table 2 and Table 3, which show that the application of the minimum potential energy principle of mixed variables to solve the problem of rectangular thin plate bending is correct.

\section{Conclusion}

In this paper, the minimum potential energy principle of the mixed variable is used to solve the problem of the bending of the cantilever rectangular thin plate with two hanging corners, one fixed edge and three free edges under the uniform load. The deflection surface equation and the calculation result are given and compared with the finite element results. It is proved that the solution of the minimum potential energy principle of mixed variable is very effective and the result given is accurate. Therefore, the minimum potential energy principle of the mixed variable provides a new method for the solution of the elastic mechanics problem, which can be applied directly to the engineering practice.

\section{References}

[1] Fu Baolian: Energy principle in elastic mechanics and its application. Science Press,Beijing( 2004),p6-56.

[2] Fu Baolian.:A new theory of bending thin plate work. Science Press, Beijing(2003),p 313-328.

[3] Hu Haichang: Variational principle of elastic mechanics and its application. Science Press, Beijing(1978) p139-153.

[4] C.M. Wang. I.M. Nazmul, T. Matsumoto. Bending Solutions of Sectorial Thick Plates Based on Reissner Plate Theory. Applied Mechanics,2005,33(1):51-77.

[5] Fu BaoLian Chen YingJie Liu HongMin. Spring back variational principle of beam with large deflection.The Third international Conference on Advanced Forming and Die Manufacturing 
Technology. 4-6 September, in Buasan, Korea(2006). 